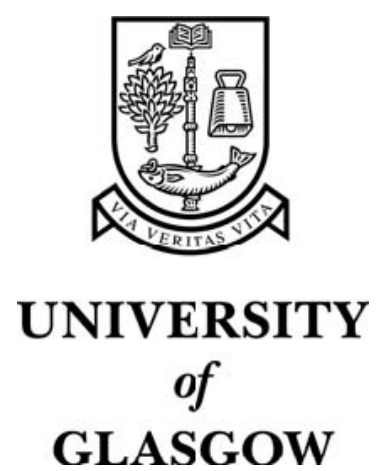

Hale, J.G. and Pollick, F.E. (2005) "Sticky Hands": learning and generalization for cooperative physical interactions with a humanoid robot. IEEE Transactions on Systems, Man and Cybernetics Part C. 35(4):pp. 512-521.

http://eprints.gla.ac.uk/3506/ 


\title{
"Sticky Hands": Learning and Generalization for Cooperative Physical Interactions With a Humanoid Robot
}

\author{
Joshua G. Hale and Frank E. Pollick
}

\begin{abstract}
Sticky Hands" is a physical game for two people involving gentle contact with the hands. The aim is to develop relaxed and elegant motion together, achieve physical sensitivityimproving reactions, and experience an interaction at an intimate yet comfortable level for spiritual development and physical relaxation. We developed a control system for a humanoid robot allowing it to play Sticky Hands with a human partner. We present a real implementation including a physical system, robot control, and a motion learning algorithm based on a generalizable intelligent system capable itself of generalizing observed trajectories' translation, orientation, scale and velocity to new data, operating with scalable speed and storage efficiency bounds, and coping with contact trajectories that evolve over time. Our robot control is capable of physical cooperation in a force domain, using minimal sensor input. We analyze robot-human interaction and relate characteristics of our motion learning algorithm with recorded motion profiles. We discuss our results in the context of realistic motion generation and present a theoretical discussion of stylistic and affective motion generation based on, and motivating cross-disciplinary research in computer graphics, human motion production and motion perception.
\end{abstract}

Index Terms-Compliance, cooperation, humanoid robots, motion generalization, motion learning, Sticky Hands.

\section{STICKY HANDS}

T HE "Sticky Hands" game was drawn from Tai Chi practice. Some schools of Tai Chi include physical contact exercises with a partner. Gentle contact is formed with a partner and maintained while moving in order to develop the facility to perform relaxed and graceful motion, leading to a sensitivity to the forces transmitted via contact. When one partner yields the other must push, and vice versa. Prolonged practice reveals the development of intuition and automatic response such that the contact may be preserved with very little force throughout a complicated and spontaneous sequence of movements. The game includes becoming comfortable with physical contact, and

Manuscript received August 6, 2001; revised September 23, 2003, March 4, 2004, and June 1, 2004. This work was supported in part by the British Council and in part by the Japan International Science and Technology Exchange Center (JISTEC). This paper was recommended by Associate Editor C. C. White.

J. G. Hale is with the the Japan Science and Technology Agency International Cooperative Research Project (JST-ICORP) Computational Brain Project, Department of Humanoid Robotics and Computational Neuroscience, ATR Computational Neuroscience Laboratories, Kyoto 619-0288, Japan. (e-mail: mail@ joshhale.com)

F. E. Pollick is with the Department of Humanoid Robotics and Computational Neuroscience, ATR Computational Neuroscience Laboratories, Kyoto 619-0288, Japan. He is also with the Deptartment of Psychology, University of Glasgow, Glasgow G12 8QB, U.K. (e-mail: frank@psy.gla.ac.uk).

Digital Object Identifier 10.1109/TSMCC.2004.840063

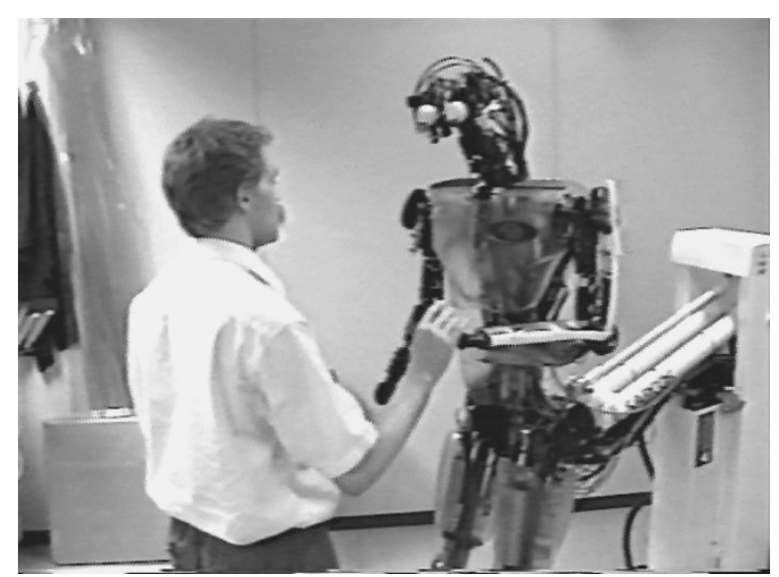

Fig. 1. Playing Sticky Hands with a humanoid robot.

a mutual goal of personal development, as well as being fulfilling and calming so it is considered by some as a form of spiritual development. An expert may play with a beginner and encourage graceful and rewarding movements by breaking down the tension in the student's motion.

Our goal was to have a humanoid robot play the game with a human partner. We defined a specific variant of the game involving contact between the hands. Partners face each other while standing and both raise one hand to their partner's. The game always begins with slow circling motions. The motion may soon diverge into a spontaneously developing trajectory as the partners explore their range of physical expression. Our robot DB may be seen playing the game in Fig. 1 (see also Fig. 2).

As a novel application it presents several interesting challenges for the design of an intelligent system, and in particular to path planning. The robot must be capable of moving while remaining compliant to contact forces from a human and be able to learn from and mimic the motion patterns of humans playing the game. By imitating the patterns produced by humans, the robot may reflect a human's creativity and encourage people to explore their range of motion. In order to do this while simultaneously experiencing new and unpredictably developing motion patterns our learning algorithm is capable of generalizing what it has seen to new conditions and maintains a suitably evolving internal state.

The motivation for this work was to explore physical interactions between humans and robots. This was inspired by the observation that the arising problem for the design of appropriate intelligent systems is how to communicate and cooperate with people [1]. Based on the principle that physical interaction is a familiar and reliable method for most humans, Sticky Hands 


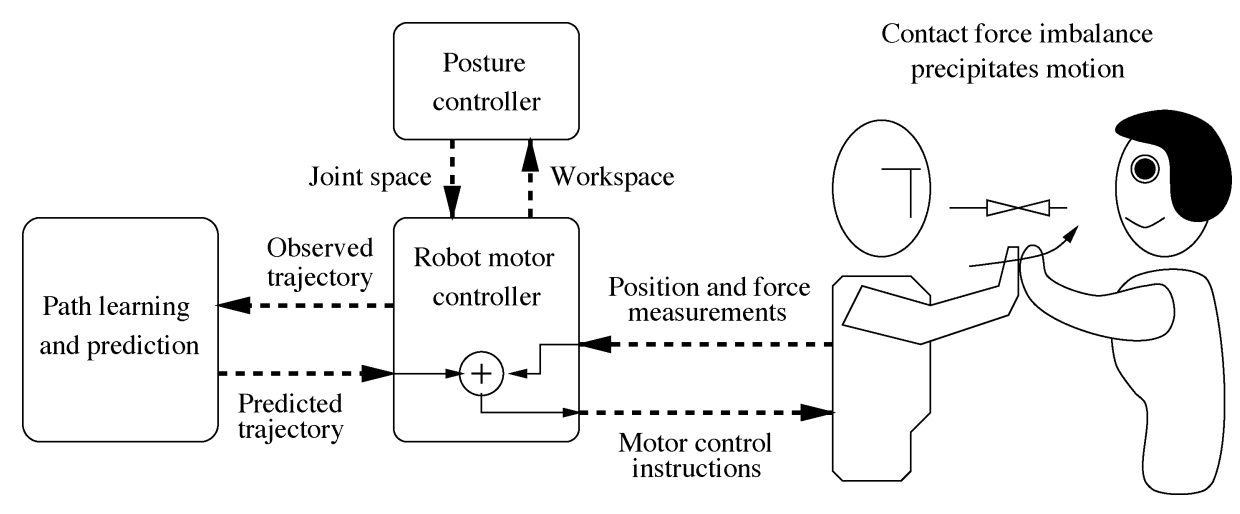

Fig. 2. System breakdown.

broaches the area of physical cooperation and the subtle but significant issue of communication [2] through physical movement [3], [4]. Our work therefore considers human imitation [5], which we consider to be of particular relevance for the future; as computer science and robotics develop it becomes clear that humans and robots will cooperate with a wide range of tasks. Furthermore, we explored the use of a humanoid robot as a playmate facilitating a human's self-development. As such the robot may assume a new social role involving a physically intimate and cooperative interaction. Hopefully, through the interaction people will be encouraged to consider the robot as a humanoid rather than mechanical entity, thus revealing ways of making human and robot interactions more natural.

Humanoid robotics captures a certain fascination with creating a mechanical entity analogous to our human selves. Other valid motivations exist [6], not least among which is our environment-being highly adapted to human sensory and motor capabilities it begs for artificial agents with analogous capabilities that can usefully coexist in our own living and working spaces. Moreover, anthropomorphic shape and organic motion makes working with such robots aesthetically and emotionally more pleasing. Of particular relevance are the production of human-like, and emotionally expressive styles of movement. The sticky hands interaction embodies human like motion and autonomy. The goal of maintaining minimal contact force is tangible while the creative, anticipatory aspect is enhanced by initiative. These challenges motivated the development of a highly generalized learning algorithm, and a theoretical investigation of expressive motion styles.

We first present a system overview that describes the relationship between robot control and learning in Section II. We then describe robot control in Section III, presenting additional sensing technology and explaining how we achieved hand placement that was compliant to external forces. Following this, we discuss the learning algorithm in Section IV which observed trajectories of the hand throughout interaction with a human and predicted the development of a current trajectory. In Section V we present results taken from an interaction between human and humanoid. In Section VI, we develop a discussion of humanoid motion synthesis and illustrate the relevance of human motion production and perception research to the generation of motion embodying emotion and style. This discussion broadens the context of the Sticky Hands exercise and motivates future cross-disciplinary research. We conclude with a brief summary of our work in Section VII.

\section{SYSTEM OVERVIEW}

The robotic Sticky Hands control system was divided into three components. Fig. 2 shows a "Robot motor controller." The motor controller is responsible for hand positioning and obeys a trajectory plan supplied by the learning algorithm. It also estimates the contact force with the human and adjusts the trajectory plan to compensate for contact force discrepancies. The hand position was sampled and this somewhat noisy data was smoothed and sent back to the learning algorithm.

The learning algorithm output predicts the hand trajectory and processes the observed trajectories supplied by the motor control. The learning algorithm observes the evolution of the hand trajectory continuously. It learns motion patterns, and generalizes them to predict future developments in the hand trajectory. The input and output are both sequences of position vectors. The robot controller makes use of the "posture controller." The posture controller used a straightforward inverse kinematics routine to generate joint configurations satisfying Cartesian hand placement targets.

The ethos of our motion system may be contrasted with the work of Williamson [7] whose motion controllers were based on positional primitives. A small number of postures were interpolated to produce target joint angles and hence joint torques according to proportional gains. The work advocated the concept of "behaviors or skills as coarsely parameterised atoms by which more complex tasks can be successfully performed," an approach also proposed for computer animation in works such as the motion verbs and adverbs of Rose et al. [8]. Williamson's system is elegant, providing a neatly bounded workspace, but not suitable for our needs since we require a continuous interaction combined with more precise positioning of the robot's hand.

\section{ROBOT CONTROL}

The 30-degree of freedom (DOF) SARCOS ${ }^{1}$ anthropomorphic robot [9] that may be seen in Fig. 3 performed the Sticky Hands exercise. It is hydraulically powered at every joint, with joint angle and joint load sensors. The low level controller positions joints by applying gain torques proportional to the angular offset between the measured and target angles, and negatively proportional to the angular velocity at each joint independently. This yields proportional gains/spring-damper control at

${ }^{1}$ SARCOS is an advanced robotics company based in Salt Lake City, UT. 

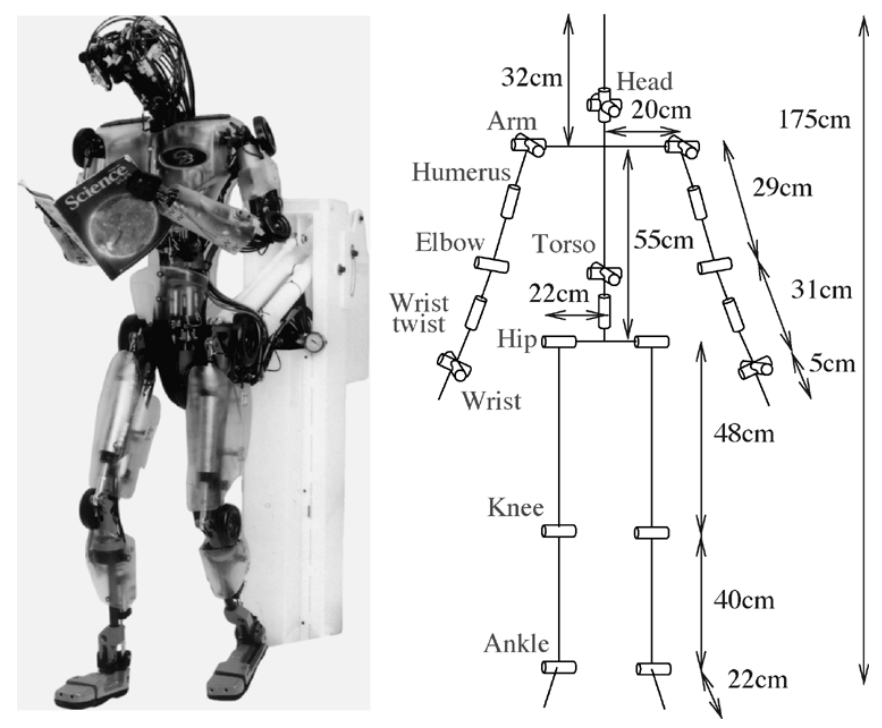

Fig. 3. DB kinematics.

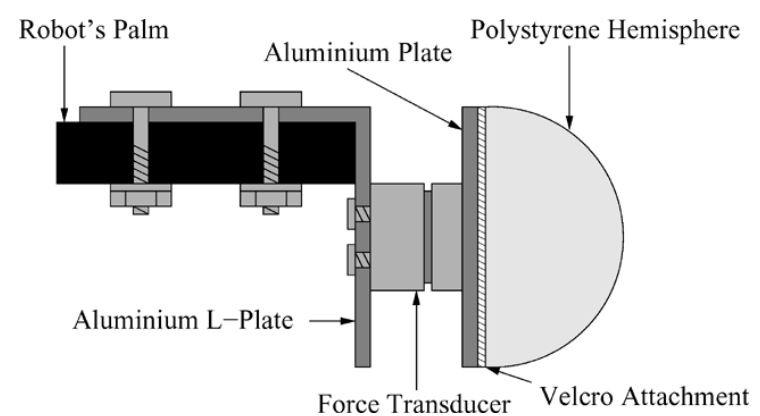

Fig. 4. Force transducer attachment.

each joint, where the torque at a given joint may be calculated as

$$
\tau=k_{s}\left(\theta_{t}-\theta\right)+k_{d}\left(\dot{\theta}_{t}-\dot{\theta}\right)
$$

where $\theta_{t}$ and $\dot{\theta}_{t}$ are the target angle and angular velocity (the latter is usually 0 ), $\theta$ and $\dot{\theta}$ are the current angle and angular velocity, and $k_{s}$ and $k_{d}$ are the spring stiffness and damping parameters, respectively.

An inverse dynamics algorithm was available to estimate the torques necessary to hold a position, and was used to reduce the magnitude of the oscillations caused by the proportional gains controller. Since the robot is anchored off the ground by its pelvis, there is not an issue with standing and balancing. The Sticky Hands exercise involves only one hand so there is a chain of 10 significant DOFs from the anchor point the robot's hand. The chain is kinematically redundant, so an iterative inverse kinematics algorithm was used [10] which resolved the unused 20 DOFs according to a default posture.

We required the robot to balance a force against its hand applied by the human player. A given trajectory was performed with adjustments to balance the contact force, making the hand actively compliant to changes of the contact force. The simplest method of determining the contact force is by means of a force transducer between the robot's and human's hands. Fig. 4 shows the attachment of the force transducer between the robot's hand and a polystyrene hemisphere intended to facilitate an ergonomic surface for the human to contact. The transducer measured forces in the $X, Y$, and $Z$ directions. We did not take torque readings from the transducer. If the contact force exceeded a 5-N threshold the target position of the hand was translated to compensate. In order to balance a force applied by the human, we subtracted a small quantity in the $Z$ (forward-backward) direction from the measured force prior to thresholding. We used this method, but also implemented a method of responding to the contact force using only the sensors internal to the SARCOS robot, i.e., joint load and angle. In both cases we assumed that the interactions were not so forceful as to necessitate more than a constant adjustment in hand position, i.e., continuous pressure would result in a constant velocity yielding movement.

One such method of calculating the externally applied force is to measure joint angles, use inverse dynamics to estimate the torques necessary to hold the position and subtract the measured loads. A discrepancy should reveal torques due to external loads other than gravity. Unfortunately inaccuracies in the dynamic model and load sensors necessitated a large contact force to facilitate suitable thresholding of the results. Instead we measured the positional offset between target and actual hand positions, assuming any large discrepancy was caused by external nongravity forces. We were able to threshold by $2 \mathrm{~cm}$ (yielding an effective force threshold of about $12 \mathrm{~N}$ ). Thus, when the controller followed a suggested trajectory the hand was directed to $5 \mathrm{~cm}$ further forward from the suggested position. This method did not require readings from the joint load sensors to respond to external forces because physical interactions were registered purely through the position of the robot's hand-which requires only joint angle readings to compute.

It was assumed that the human would supply a force sufficient to maintain the robot's hand at the suggested position, opposing the $5 \mathrm{~cm}$ offset. If the human reduced or increased the contact force the robot's hand would move beyond the $2 \mathrm{~cm}$ threshold and the suggested trajectory consequently adjusted to compensate. While the contact force was entirely in the $Z$ direction, any $X$ and $Y$ as well as $Z$ perturbations would be accommodated when the threshold was reached. By using this indirect kinematic method we facilitated the use of a significantly lighter contact force than with inverse dynamics based force estimation. In fact, any reasonable positioning and compliance strategy is possible. In the ideal case the force threshold is minimal, since the larger the threshold the higher the necessary contact force and consequently the less relaxed the human's motion becomes. The kinematics of robot limb placement do not significantly affect the contact force but may affect the perception of the robot motion, as we discuss in Section VI. Section V compares this kinematic technique with the force transducer method and presents traces of the forces measured during interaction with a human.

Trajectories output by the learning algorithm were described using piecewise linear splines. The robot controller ran at $500 \mathrm{~Hz}$ and the learning algorithm ran at $10 \mathrm{~Hz}$. The sequence of predictions output by the learning algorithm were interpreted as the advancing end-point of a spline. If the motor controller detected a discrepancy in the contact force, the knots were translated to compensate. The translated knots were smoothly 


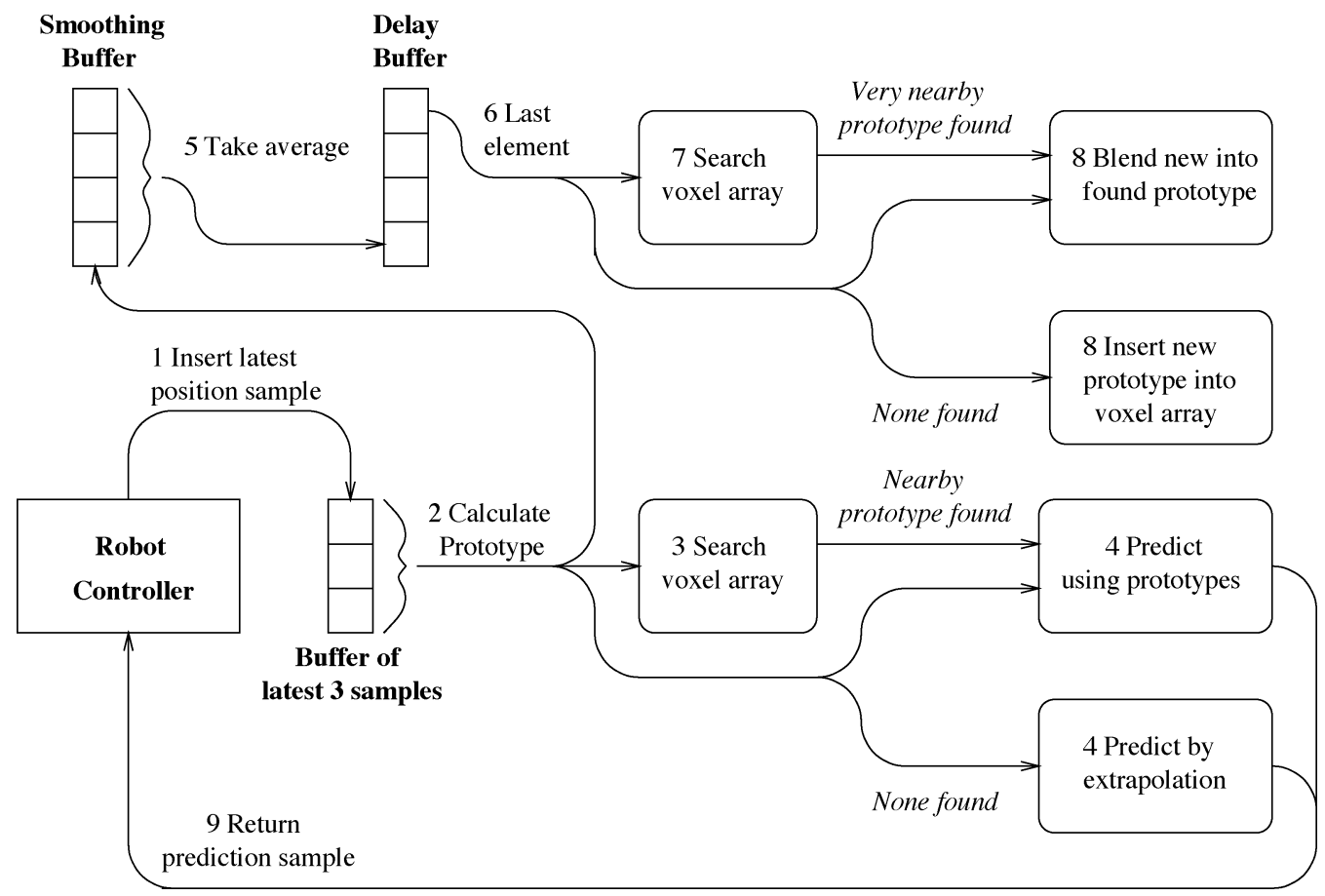

Fig. 5. Learning algorithm.

decayed back to their position prior to the compensatory translation using a smoothing vector. The smoothing vector was initialized with the translation vector and decayed, tending to zero, during every cycle of the motor controller. This smoothing prevented the hand from jerking in response to contact forces, instead smoothly moving to accommodate knot translations.

\section{IV. "PRototype SET” LEARNING AlgORITHM}

The learning algorithm received 3-D point samples describing the robot's hand trajectory. For each sample, a vector predicting the progression of the trajectory was output. The nature of the Sticky Hands exercise with respect to a computer implementation demanded the following requirements, fulfilled by our algorithm.

- Generalize observed trajectories for prediction of similar new trajectories with different orientation, scale, curvature, position or velocity.

- Extrapolate properties of a new trajectory for prediction in the absence of similar observed trajectories.

- Fluid internal state copes with the evolving nature of trajectories through continuous update, replacement and "forgetting" of recorded information.

- Branch points where similar observed trajectories diverge are not problematic.

- Noise causing inaccurate position samples is not problematic.

- Parameterisable time bound ensures real time operation.

- Parameterisable memory bound facilitates exploitation of host architecture.

The algorithm recorded instantaneous properties of the input trajectory in structures we refer to as a "prototypes." By comparison to the work of Stokes et al. [11] who presented a method for identifying cyclic patterns and their significance in space-line samples, our process focuses on the immediate instant of a trajectory. Salient features are recorded for efficient retrieval but no internal classifications of higher level structures such as cycles are made. This is the essence of the generalization and branch point handling properties of our algorithm since the recorded properties of any instant of an observed trajectory may be used to predict the development of any new trajectory, and no information about correlations between trajectories is explicitly maintained by the prediction process.

Below we define the "prototype" mathematically, and demonstrate how it can be used for prediction or extrapolation. We then show how the most appropriate prototype for predicting a given trajectory may be selected from a memory bank by means of a distance metric between prototypes and an optimized search procedure. The creation of prototypes from raw position data is explained, followed by a reinforcement memory technique designed to ensure an efficient use of memory. The reader may find it useful to refer to Fig. 5 throughout this prototype learning section.

\section{A. Prediction Using Prototypes}

Suppose we describe the sequence of input position samples as $\left\{p_{k}: k \in \mathbf{N}\right\}$. The prototype $P_{i}$ corresponding to $p_{i}$ is defined using $p_{i-1}, p_{i}$ and $p_{i+1}$

$$
\begin{aligned}
P_{i} & =\left(p_{i}, v_{i}, a_{i}, T_{i}\right) \\
v_{i} & =p_{i+1}-p_{i} \in \mathbf{R}^{3} \\
a_{i} & =\frac{\left|p_{i+1}-p_{i}\right|}{\left|p_{i}-p_{i-1}\right|} \in \mathbf{R} \\
T_{i} & =\left(\cos \left(\frac{\theta_{i}}{2}\right), \sin \left(\frac{\theta_{i}}{2}\right)\left(p_{i+1}-p_{i}\right) \times\left(p_{i}-p_{i-1}\right)\right) \\
\theta_{i} & =\cos ^{-1}\left(\frac{\left(p_{i}-p_{i-1}\right) \cdot\left(p_{i+1}-p_{i}\right)}{\left|p_{i}-p_{i-1}\right|\left|p_{i+1}-p_{i}\right|}\right) \in \mathbf{R}
\end{aligned}
$$


Previously observed trajectory

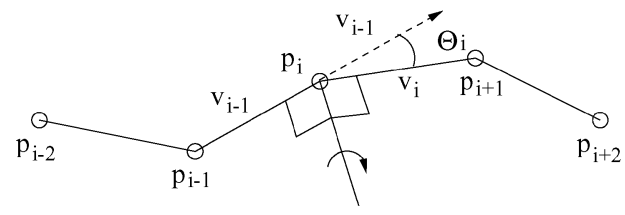

Prediction

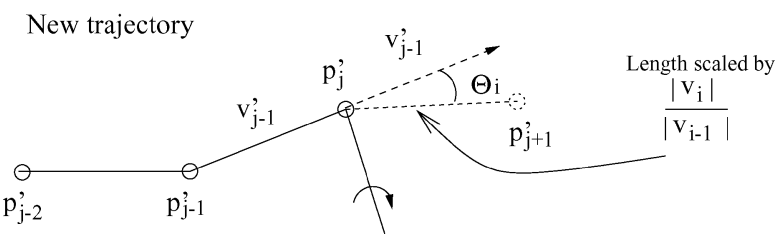

Fig. 6. Trajectory prediction using a prototype.

where $v_{i}$ is the velocity out of $p_{i}$ (scaled by an arbitrary factor) and $a_{i}$ is a scalar indicating the magnitude of the acceleration. The acceleration direction is deducible from $T_{i}$, a quaternion describing the change in direction between $v_{i}$ and $v_{i-1}$ as a rotation through their mutually orthogonal axis.

The progression of a trajectory $\left\{p_{k}^{\prime}: k \in \mathbf{N}\right\}$ may be predicted using a prototype. Suppose initially, that it is known that $P_{i}$ corresponds to $p_{j}^{\prime}$, then $p_{j}^{\prime}+a_{i} T_{i}\left(p_{j}^{\prime}-p_{j-1}^{\prime}\right)$ is an estimate for $p_{j+1}^{\prime}$. Premultiplication of a three-vector by $T_{i}$ denotes quaternion rotation in the usual way. This formula applies the bend and acceleration occurring at $p_{i}$ to predict the position of $p_{j}^{\prime}$. We also linearly blend the position of $p_{i}$ into the prediction, and the magnitude of the velocity so that $p_{j}^{\prime}$ combines the actual position and velocity of $p_{i}$ with the prediction duplicating $p_{i}$ 's bending and accelerating characteristics (see Fig. 6)

$$
\begin{aligned}
p_{j+1}^{\prime} & =p_{j}^{\prime}+s_{j} T_{i} \cdot \frac{p_{j}^{\prime}-p_{j-1}^{\prime}}{\left|p_{j}^{\prime}-p_{j-1}^{\prime}\right|}+g_{\mathrm{p}}\left(p_{i}-p_{j}^{\prime}\right) \\
s_{j} & =\left(1-g_{\mathrm{v}}\right) a_{i}\left|p_{j}^{\prime}-p_{j-1}^{\prime}\right|+g_{\mathrm{v}}\left|v_{i}\right| .
\end{aligned}
$$

The blending ratios $g_{\mathrm{p}}$ and $g_{\mathrm{v}}$ are used to manage the extent to which predictions are entirely general, or repeat previously observed trajectories. i.e., how much the robot wants to repeat what it has observed. We chose values of $g_{\mathrm{p}}$ and $g_{\mathrm{v}}$ in the range [00.1, $0.001]$ through empirical estimation. $g_{\mathrm{p}}$ describes the tendency of predictions to gravitate spatially toward recorded motions, and $g_{\mathrm{v}}$ has the corresponding effect on velocity.

In the absence of a corresponding prototype we can calculate $P_{j-1}^{\prime}$, and use it to estimate $p_{j+1}^{\prime}$, thus extrapolating the current characteristics of the trajectory. Repeated extrapolations lie in a single plane determined by $p_{i-2}, p_{i-1}$ and $p_{i}$. This is not problematic because when guided into a new motion pattern we only require the robot to move in a sensible way so that the human may continue to guide it. We must set $g_{\mathrm{p}}=0$ since positional blending makes no sense when extrapolating, and would cause the trajectory to slow to a halt, i.e., the prediction should be based on an extrapolation of the immediate velocity and turning of the trajectory and not averaged with its current position since there is no established trajectory to gravitate toward.

\section{B. Storage and Retrieval}

The prediction of $p_{j+1}^{\prime}$ requires ideally that an observed trajectory with similar characteristics to that observed at $p_{j}^{\prime}$ is found. Given a set of recorded prototypes it is necessary to find a prototype $P_{i}$ to be used as described above for prediction. The prototype $P_{j-1}^{\prime}$ describing the current trajectory can be used as a basis for identifying similar prototypes corresponding to similar, previously observed trajectories We define a distance metric relating prototypes in order to characterize the closest match

$$
d\left(P_{i}, P_{j}\right)=1-\cos \left(\theta^{\prime}\right)+\frac{\left|p_{i}-p_{j}\right|}{M_{\mathrm{p}}}
$$

where

$$
\begin{aligned}
\theta^{\prime} & =\theta \frac{\pi}{2 M_{a}}, \quad \theta \in\left[-M_{a}, M_{a}\right] \\
& =\pi, \quad \text { otherwise } \\
\theta & =\cos ^{-1} \frac{v_{i} \cdot v_{j}}{\left|v_{i}\right|\left|v_{j}\right|} .
\end{aligned}
$$

$M_{a}$ and $M_{\mathrm{p}}$ define the maximum angular and positional differences such that $d\left(P_{i}, P_{j}\right)$ may be one or less. Prototypes within this bound are considered similar enough to form a basis for a prediction. In practice, the values of $15 \mathrm{~cm}$ and $\pi / 4$ for $M_{\mathrm{p}}$ and $M_{a}$, respectively, were found to be suitable. The values must be large enough to permit some generalization of observed trajectories, but no so large that totally unrelated motions are considered suitable for prediction.

The metric compares the position of two prototypes, and the direction of their velocities. Two prototypes are close if they describe a trajectory traveling in the same direction, in the same place. The absolute velocity, and bending characteristics are not compared. Predictions are therefore general with respect to the path leading a trajectory to a certain position with a certain direction (so branching points are not problematic) and velocity, so the speed at which an observed trajectory was performed does not affect the way it can be generalized to new trajectories. This applies equally to the current trajectory and previously observed trajectories.

When seeking a prototype we might compare all recorded prototypes with $P_{j-1}^{\prime}$ to find the closest. If none exist within a distance of 1 we use $P_{j-1}^{\prime}$ itself to extrapolate as above. Needless to say we could not compare $P_{j-1}^{\prime}$ with all the recorded prototypes. To optimize this search procedure we defined a voxel array to store the prototypes. The array encompassed a cuboid enclosing the reachable space of the robot, partitioning it into a $50 \times 50 \times 50$ array of cuboid voxels indexed by three integer coordinates. The storage requirement of the empty array was $0.5 \mathrm{Mb}$. New prototypes were placed in a list attached to the voxel containing their positional component $p_{i}$. Given $P_{j-1}$ we only needed to consider prototypes stored in voxels within $M_{\mathrm{p}}$ of $p_{j-1}$ since prototypes in any others would definitely exceed the maximum distance according to the metric.

The order in which voxels were considered was optimized by preparing a list of voxel index offsets. The list was sorted by minimum distance offset and was cropped at the maximum distance $M_{p}$. This ensures an optimal search of the voxel array since the voxels are considered in an expanding sphere about the voxel containing the original prototype, and the search may terminate as soon as we encounter a voxel that is too far away to contain a prototype with a closer minimum distance than any 
already found. It also permits the search to be cut short if time is unavailable. In this case the search terminates optimally since the voxels most likely to contain a match are considered first. This facilitates the parameterisable time bound since the prototype search is by far the dominant time expense of the learning algorithm.

\section{Creation and Maintenance}

Prototypes were continually created based on the input position samples describing the observed trajectory. It was possible to create a new prototype for each new sample, which we placed in a cyclic buffer. For each new sample we extracted the average prototype of the buffer to reduce sampling noise. A buffer of 5 elements was sufficient. The averaged prototypes were shunted through a delay buffer, before being added to the voxel array. This prevented prototypes describing a current trajectory from being selected to predict its development (extrapolation) when other prototypes were available. The delay buffer contained 50 elements, and the learning algorithm was iterated at $10 \mathrm{~Hz}$ so that new prototypes were delayed by $5 \mathrm{sec}$.

Rather than recording every prototype we limited the total number stored by averaging certain prototypes. This ensures the voxel array does not become clogged up and slow, and reduces the memory requirement. Therefore before inserting a new prototype into the voxel array we first searched the array for a similar prototype. If none was found we added the new prototype, otherwise we blended it with the existing one. We therefore associated a count of the number of blends applied to each prototype to facilitate correct averaging with new prototypes. In fact we performed a nonlinear averaging that capped the weight of the existing values, allowing the prototypes to tend toward newly evolved motion patterns within a limited number of demonstrations. Suppose $P_{a}$ incorporates $n$ blended prototypes, then a subsequent blending with $P_{\mathrm{b}}$ will yield

$$
\begin{aligned}
P_{a^{\prime}} & =P_{a} \frac{D(n)-1}{D(n)}+P_{\mathrm{b}} \frac{1}{D(n)} \\
D(n) & =1+A_{\mathrm{M}}-\frac{A_{\mathrm{M}}}{1+n A_{\mathrm{G}}}
\end{aligned}
$$

where $A_{\mathrm{M}} /\left(1+A_{\mathrm{M}}\right)$ defines the maximum weight for the old values, and $A_{\mathrm{G}}$ determines how quickly it is reached. Values of 10 and 0.1 for $A_{M}$ and $A_{G}$, respectively, were found to be suitable. This makes the averaging process linear as usual for small values but ensures the contribution of the new prototype is worth at least $1 / 11$ th.

We facilitated an upper bound on the storage requirements using a deletion indexing strategy for removing certain prototypes. An integer clock was maintained, and incremented every time a sample was processed. New prototypes were stamped with a deletion index set in the future. A list of the currently stored prototypes sorted by deletion index was maintained, and if the storage bounds were reached the first element of the list was removed and the corresponding prototype deleted. The list was stored as a heap [12] since this data structure permits fast $O(\log ($ Num Elements $))$ insertion, deletion and repositioning. We manipulated the deletion indexes to mirror the reinforcement aspect of human memory. A function $R(n)$ defined the period for which a prototype reinforced $n$ times should be retained
( $n$ is equivalent to the blending count). Each time a prototype was blended with a new one we calculated the retention period, added the current clock and re-sorted the prototype index. $R(n)$ increases exponentially up to a maximum asymptote

$$
R(n)=D_{\mathrm{M}}-\frac{D_{\mathrm{M}}}{1+D_{\mathrm{G}} n^{D_{\mathrm{P}}}} .
$$

$D_{\mathrm{M}}$ gives the maximum asymptote. $D_{\mathrm{G}}$ and $D_{\mathrm{P}}$ determine the rate of increase. Values of 20000, 0.05, and 2 were suitable for $D_{M}, D_{G}$ and $D_{P}$, respectively. The initial reinforcement thus extended a prototype's retention by $2 \mathrm{~min}$, and subsequent reinforcements roughly doubled this period up to a maximum of about half an hour (the algorithm was iterated at $10 \mathrm{~Hz}$ ).

\section{RESUlts}

Fig. 7 shows the position of recorded prototypes for the default initial state and a stored state after a human has played with the robot for a few minutes. The two data sets are each viewed from two directions. The units in this and subsequent figures are millimeters. The coordinates are in millimeters, with the $X, Y$, and $Z$ axes positive in the robot's left, up and forward directions, respectively. The point $(0,0,0)$ corresponds to the robot's sacrum. Please observe that the robot icons illustrate orientation only, and not scale. Each point represents a prototype stored in the motion predictor's memory. The trajectory of the hand loosely corresponds to the spacing of prototypes but not exactly because sometimes new prototypes are blended with old prototypes according to the similarities between each's position and velocity vectors.

The initial state was taught to the robot and approximates a circle $10 \mathrm{~cm}$ in radius and centred in front of the left elbow joint (when the arm is relaxed) in the frontal plane about $30 \mathrm{~cm}$ in front of the robot. The prototypes correspond to the robot's left hand, which was in contact with the human's right hand. The changes in the trajectory mostly occur gradually as human and robot slowly develop repeated cycles. Once learned, the robot can switch between any previously performed trajectories.

In order to assess the effect of blending motions planned by the prediction algorithm with this compliance system we compare the Sticky Hands controller with a "positionable hand" controller that only maintains a fixed target for the hand in a compliant manner so that a person may reposition the hand. Fig. 8 shows a force/position trace where the width of the line is linearly proportional to the magnitude of the force vector (measured in all three dimensions), and Table I shows corresponding statistics. Force measurements are averaged over a one minute period of interaction, but also presented are "complied forces," averaging the force measurements over only the periods when the measured forces exceeded the compliance threshold. From these results it is clear that using the force transducer yielded significantly softer compliance in all cases. Likewise the "positionable hand" task yielded slightly softer compliance because the robot did not attempt to blend its own trajectory goals with those imposed by the human.

Many of the properties of the system can be seen through examination of a sequence of interaction between the robot and human. Fig. 9 shows such a sequence during which the robot used the kinematic compliance technique. The motion is in a 


\section{Initial state}
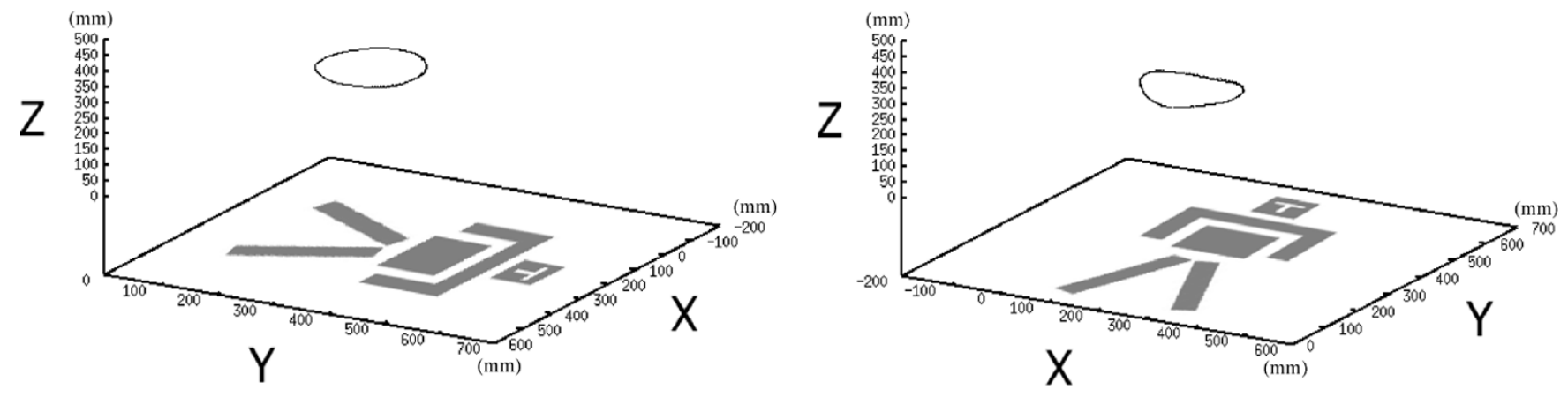

\section{Human interaction}

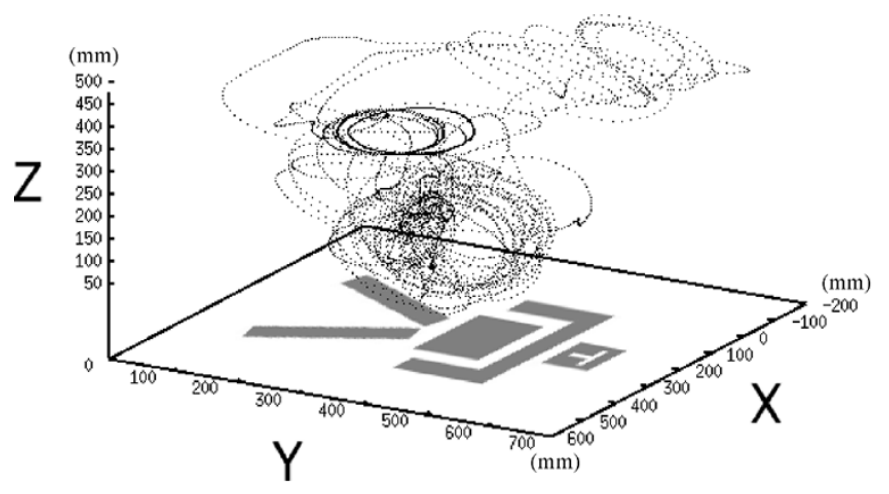

Fig. 7. Prototype state corresponding to a sample interaction.

clockwise direction, defined by progress along the path in the a-b-c direction, and was the first motion in this elliptical pattern observed by the prediction system. The "compliant adjustments" graph shows the path of the robot's hand, and is marked with thicker lines at points where the compliance threshold was exceeded. i.e., points where the prediction algorithm was mistaken about the motion the human would perform. The "target trajectory" graph shows in lighter ink the target sought by the robot's hand along with in darker ink the path of the robot's hand. The target is offset in the $Z$ (forward) direction in order to bring about a contact force against the human's hand. At point (a) there is a kink in the actual hand trajectory, a cusp in the target trajectory, and the beginning of a period during which the robot experiences a significant force from the human. This kink is caused by the prediction algorithm's expectation that the trajectory will follow previously observed patterns that have curved away in the opposite direction, the compliance maintaining robot controller adjusts the hand position to attempt to balance the contact force until the curvature of the developing trajectory is sufficient to extrapolate its shape and the target trajectory well estimates the path performed by the human. At point $(b)$ however, the human compels the robot to perform an elliptical shape that does not extrapolate the curvature of the trajectory thus far. At this point the target trajectory overshoots the actual trajectory due to its extrapolation. Once again there is a period of significant force experienced against the robot's hand and the trajectory is modified by the compliance routine.

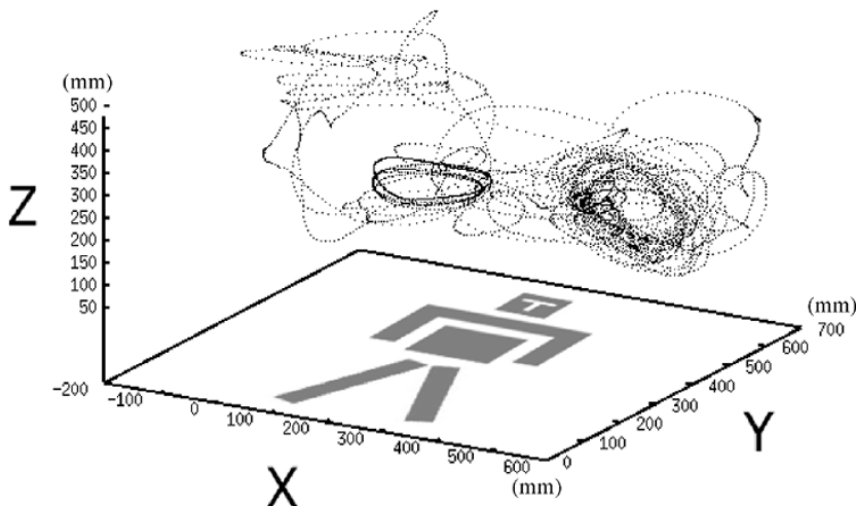

At point (c) we observe that, based on the prototypes recorded during the previous ellipse, the prediction algorithm correctly anticipates a similar elliptical trajectory offset positionally and at a somewhat different angle. ${ }^{2}$

\section{DISCUSSION}

We now present a theoretical discussion of the production of motion embodying style and affect, which we believe to be a valuable perspective as well as the natural direction for extension of this work. Motion generation for the Sticky Hands application has been based entirely upon the prototype set algorithm and compliant control mechanism. However, the system was designed to be able to incorporate the augmentation of motion with style or affect, which we envisage in the "posture controller" component of the system (Fig. 2). Such motion augmentation may be inspired by research describing how humans produce and perceive movements as well as recent techniques in computer animation. For example, there is a strong duality between dynamics based computer animation and robotics [14]. Computer animation provides a rich source of techniques for generating [15]-[20] and manipulating [21] dynamically correct motion, simulating biomechanical properties of the human body [22] and adjusting motions to display affect or achieve new goals [14], [23].

${ }^{2}$ Videos of a human playing the game with the robot or another human may be downloaded from http://www.dcs.gla.ac.uk/ halej/stickyHands.htm+. 


\section{Positionable Hand}
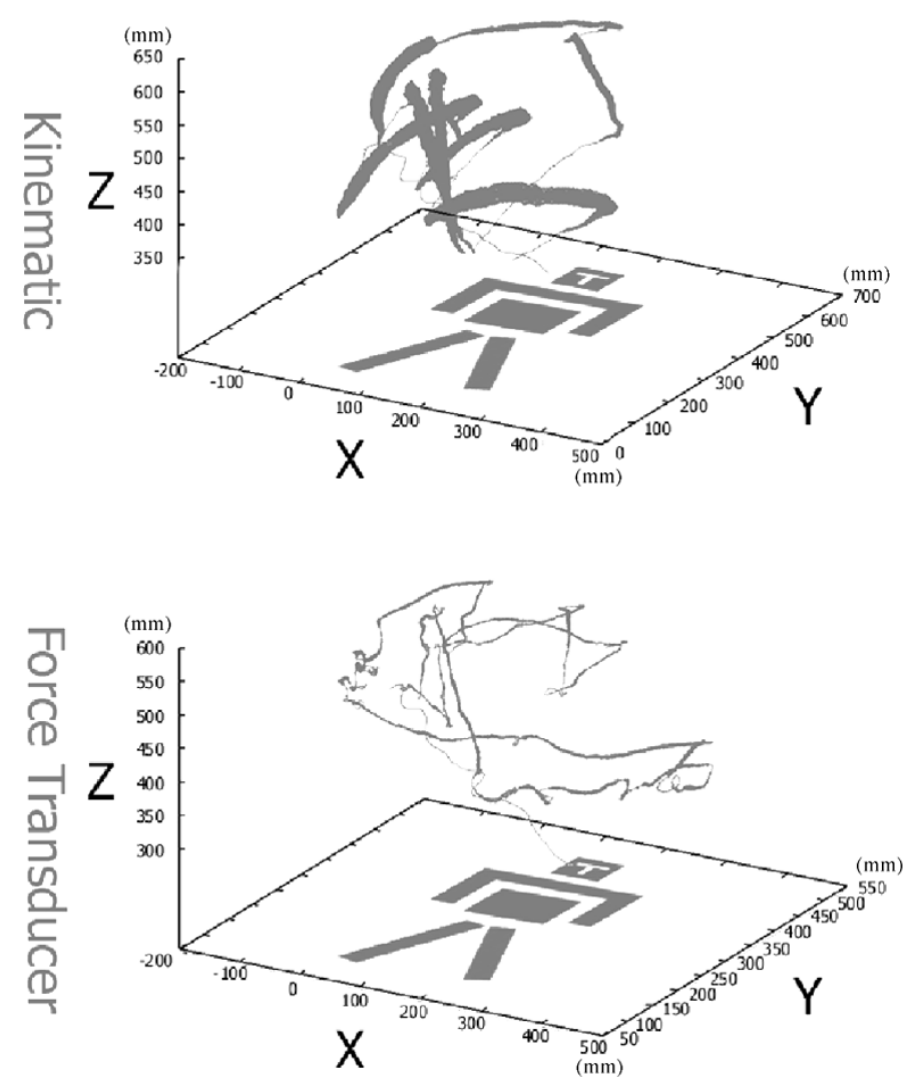
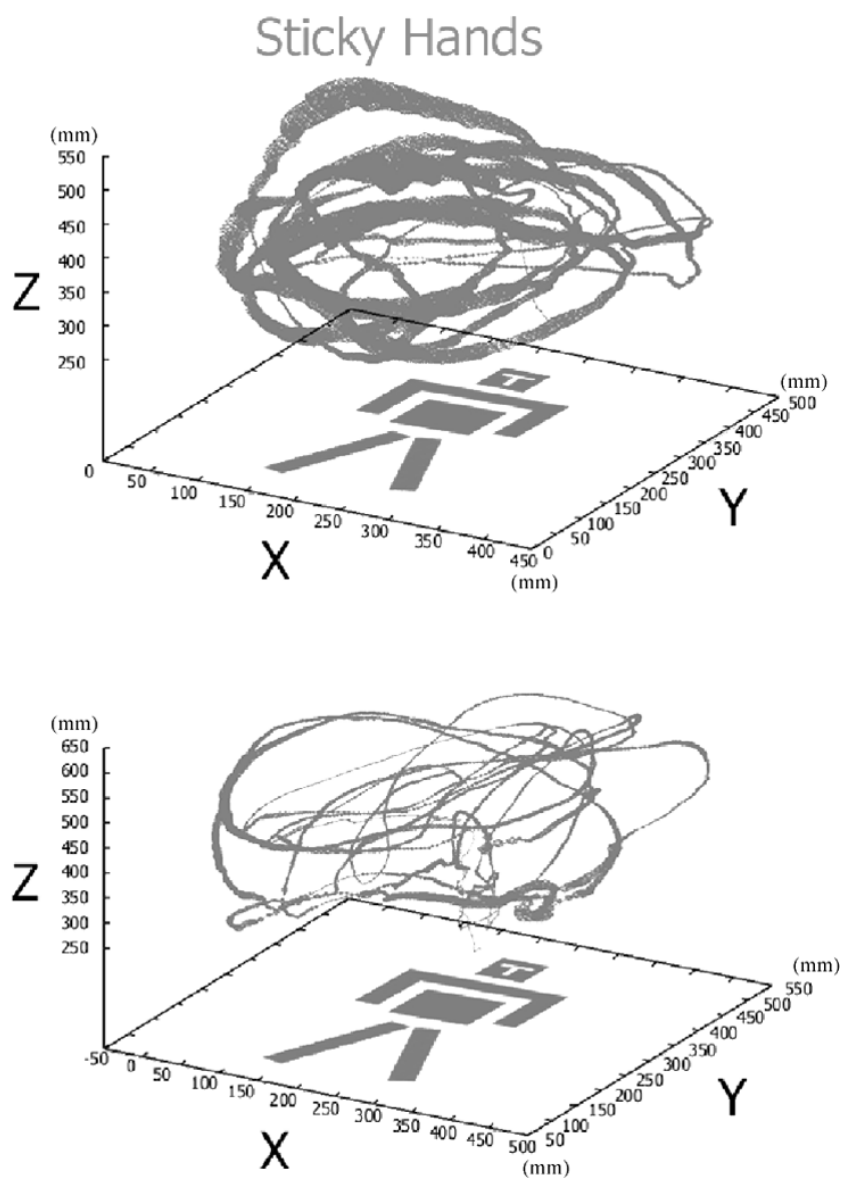

Fig. 8. Force measured during "positionable hand" and Sticky Hands tasks.

TABLE I

ForCES EXPERIENCED DURING "Positionable HAND" AND STICKY HANDS TASKS

\begin{tabular}{c|c|c|c|c}
\hline Task & \multicolumn{2}{|c|}{ Contact force $(\mathrm{N})$} & \multicolumn{2}{c}{ Complied forces (N) } \\
& Mean & Var. & Mean & Var. \\
\hline Force Transducer Sticky Hands & $\mathbf{4 . 5 0}$ & $\mathbf{4 . 8 3}$ & $\mathbf{5 . 7 2}$ & $\mathbf{4 . 4 9}$ \\
Force Transducer 'Positionable Hand' & $\mathbf{1 . 7 5}$ & $\mathbf{2 . 1 8}$ & $\mathbf{3 . 2 3}$ & $\mathbf{2 . 3 6}$ \\
Kinematically Compliant Sticky Hands & $\mathbf{1 1 . 8 6}$ & $\mathbf{1 0 . 7 3}$ & $\mathbf{1 3 . 1 5}$ & $\mathbf{1 0 . 7 3}$ \\
Kinematically Compliant 'Positionable Hand' & $\mathbf{8 . 9 0}$ & $\mathbf{1 0 . 3 8}$ & $\mathbf{1 2 . 9 3}$ & $\mathbf{1 1 . 4 0}$ \\
\hline
\end{tabular}

While the technical means to obtain natural-looking movements that show affect, skill, etc., are fundamental, it is useful to examine the production and visual perception of human movement. The study of human motor control for instance provides the potential for better techniques to mimic human movement since interactions between humans and humanoids may improve if both have similar representations of movement. For example, in the current scenario the goal is for the humanoid and the human to achieve a smooth and graceful trajectory. There are several objective ways to express smoothness and it can be anticipated that if the humanoid and human shared the same representation of smoothness then the two representations may converge more quickly on a graceful path. Study of the visual perception of human movement holds the potential to isolate the aspects of movement that are crucial for a correct interpretation. For example, although movement can generally be thought of as a complicated spatiotemporal pattern, recognition of particular styles of movement might rely on a limited spatial or temporal aspect of the movement. Knowledge of human motor control and the visual perception of human movement could greatly facilitate the design of humanoid movements.

There are several results from human motor control and motor psychophysics which contribute to our understanding of natural human movements. It is generally thought that the smoothness of human arm movements is a result not only of the low-pass filter characteristics of the musculoskeletal system, but also the use of smoothness criteria in central movement planning. Criteria which minimize the jerk [24], torque change [25], motor-command change [26], or signal dependent error [27] have all been suggested as possibilities. Besides smoothness, further regularities to human arm movements have been reported. These include that the endpoint trajectory of the hand behaves like a segmented collection [28] of piecewise planar segments joined together [29] and that movement speed is related to its geometry defined by curvature and torsion. Specifically, it has been reported that for planar segments velocity is 


\section{Compliant Adjustments}

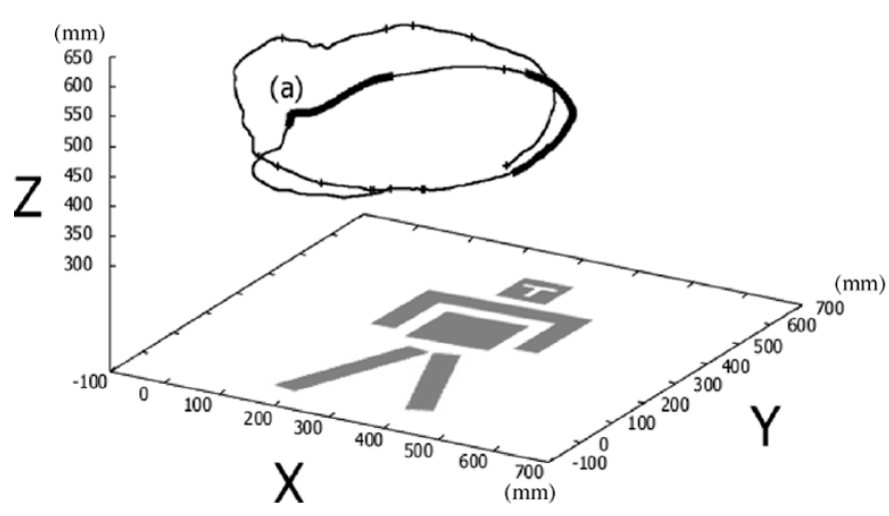

Fig. 9. Example interaction showing target trajectory and compliance activation.

inversely proportional to curvature raised to the $1 / 3$ power, and that for nonplanar segments the velocity is inversely proportional to the $1 / 3$ power of curvature multiplied by $1 / 6$ power of torsion [30]-[33]. Extensive psychological experiments of the paths negotiated by human-humanoid dyads could inform which principles of human motor control are appropriate for describing human-humanoid cooperative behaviors.

Recent results examining the visual recognition of human movement might also be relevant to the generation of various humanoid movement styles. For example, examination of the relationship between movement kinematics and style recognition have indicated how exaggeration of temporal [34], spatial [35], or spatiotemporal [36], [37] cues can be used to enhance recognition. Further evidence for kinematic specification of movement style has been found in how affect is inferred from human movement [38]. These results on how movement kinematics specify movement style not only help to constrain the design of humanoid motion, but also open the opportunity to use the humanoid robot to explore the possibility that meaningful differences in kinematics rely on the control of movement dynamics.

The brief literature review on human motor control and visual perception of human movement above provides a starting point for the design of interaction with humanoid robots. These results focus mainly on the motion of the robot and try to deal in a bottom up fashion, assuming that basic relations can be found that would indicate whether or not a movement appears natural or with the proper affect. However, it has yet to be seen that cognitive factors, in the form of expectancies and top down influences might not dominate interactions between humans and humanoids. The possibility exists that the humanoid could produce a natural movement with affect, but would be misinterpreted due to the fact that it is expected that the robot would not move naturally or display affect. Our current work is aimed at studying human-humanoid interactions at the Sticky Hands game with the bottom-up approach to the generation of natural and affective movements. However, as this work develops we anticipate an increasing role for cognitive factors in the design of humanoid interaction.

\section{Target Trajectory}

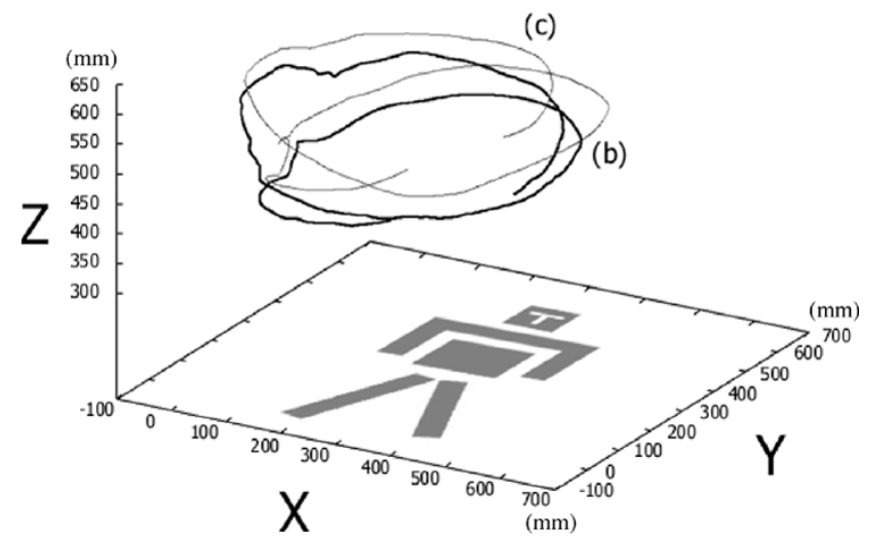

\section{CONCLUSION}

We proposed the "Sticky Hands" game as a novel interaction between human and robot. We implemented the game using a robot controller process and learning algorithm with a novel internal representation. Since our approach was nonpattern based, branching trajectories were implicitly handled without the need for segmentation analysis. Arbitrary speed and space limits could also be set by restricting the number of prototypes examined or stored. We facilitated physically intimate interactions with the humanoid robot, allowing it to take on the role of playmate and partner aiding a human's self-development. The system required minimal sensor input. Only torque and joint position sensors that one may expect to find on such a robot were required by the low level motor controller for the robot, although less forceful results were obtained with an additional force transducer. Our work may be considered as a novel communication mechanism that accords with the idea that an autonomous humanoid robot should accept command input and maintain behavioral goals at the same level as sensory input [6]. However, regarding the issue of human instruction we have demonstrated that the blending of internal goals with sensed input can yield complex behaviors that demonstrate a degree of initiative. Other contrasting approaches [13] have achieved robust behaviors that emphasize the utility of human instruction in the design of reinforcement functions or progress estimators. Finally, we have illustrated the value of cross-disciplinary research relating humanoid robotics, human motion production and motion perception by summarizing several relevant works.

\section{ACKNOWLEDGMENT}

The authors would like to thank M. Riley for much useful assistance during the development of the system, C. Atkeson for much helpful advice, S. Kotosaka for his kind and patient help with the robot control system, T. Shibata for mechanical assistance with the robot, A. Ude for his assistance with a video presentation, and S. Schaal for developing the simulation environment that facilitated the completion of this project. 


\section{REFERENCES}

[1] H. Takeda, N. Kobayashi, Y. Matsubara, and T. Nishida, "Toward ubiquitous human-robot interaction," in Proc. IJCAI Workshop on Intelligent Multimodal Systems, Nagoya, Japan, Aug. 1997, pp. 1-8.

[2] P. Coppin, R. Pell, M. D. Wagner, J. R. Hayes, J. Li, L. Hall, K. Fischer, D. Hirschfield, and W. L. Whittaker, "EventScope: amplifying human knowledge and experience via intelligent robotic systems and information interaction," in Proc. IEEE Int. Workshop on Robot-Human Interaction, Osaka, Japan, Sep. 2000, pp. 292-296.

[3] B. Adams, C. Breazeal, R. A. Brooks, and B. Scassellati, "Humanoids robots: a new kind of tool," IEEE Intell. Syst., pp. 25-31, Jul.-Aug. 2000.

[4] H. Hikiji, "Hand-shaped force interface for human-cooperative mobile robot," in Proc. 2000 IEICE General Conf., 2000, pp. 300-307.

[5] B. Scassellati, "Investigating models of social development using a humanoid robot," in Biorobotics, B. Webb and T. Consi, Eds. Cambridge, MA: MIT Press, 2000

[6] T. Bergener, C. Bruckhoff, P. Dahm, H. Janben, F. Joublin, and R. Menzner, "Arnold: an anthropomorphic autonomous robot for human environments," in Proc. Selbstorganization von Adaptivem Verhalten (SOAVE 97), Sep. 1997, pp. 25-34.

[7] M. M. Williamson, "Postural primitives: interactive behavior for a humanoid robot arm," in Proc. SAB 96, Cape Cod, MA, 1996, pp. 124-135.

[8] C. Rose, B. Bodenheimer, and M. F. Cohen, "Verbs and adverbs: multidimensional motion interpolation," IEEE Comput. Graph. Applicat., vol. 18, no. 5, pp. 32-40, May 1998.

[9] C. G. Atkeson, J. G. Hale, M. Kawato, S. Kotosaka, F. E. Pollick, M. Riley, S. Schaal, T. Shibata, G. Tevatia, A. Ude, and S. Vijayakumar, "Using humanoid robots to study human behavior," IEEE Intell. Syst., vol. 15 , no. 4 , pp. 46-56, Jul. 2000.

[10] G. Tevatia and S. Schaal, "Inverse kinematics for humanoid robots," in IEEE Int. Conf.Robotics and Automation, San Francisco, CA, Apr. 2000, pp. 294-299.

[11] V. P. Stokes, H. Lanshammar, and A. Thorstensson, "Dominant pattern extraction from 3-D kinematic data," IEEE Trans. Biomed. Eng., vol. 46, no. 1, pp. 100-106, Jan. 1999.

[12] T. H. Cormen, C. E. Leiserson, and R. L. Rivest, Introduction To Algorithms. New York: McGraw-Hill, 1990.

[13] B. Scassellati, "Knowing what to imitate and knowing when you succeed," in Proc. AISB Symp. Imitation in Animals and Artifacts, Edinburgh, U.K., April 7-9, 1999, pp. 105-113.

[14] K. Yamane and Y. Nakamura, "Dynamics filter: toward real-time and interactive motion generator for human figures," in Proc. WIRE 2000, Pittsburgh, PA, 2000, pp. 27-34.

[15] A. Witkin and M. Kass, "Spacetime constraints," in Proc. SIGGRAPH 88, Computer Graphics Annu. Conf. Series, 1988, pp. 159-168.

[16] M. F. Cohen, "Interactive spacetime control for animation," in Proc. SIGGRAPH 92, Computer Graphics Annu. Conf. Series, 1992, pp. 293-302.

[17] J. T. Ngo and J. Marks, "Spacetime constraints revisited," in Proc. SIGGRAPH 93, Computer Graphics Annu. Conf. Series, 1993, pp. 343-350.

[18] Z. Li, S. J. Gortler, and M. F. Cohen, "Hierarchical spacetime control," in Proc. SIGGRAPH 94, Computer Graphics Annu. Conf. Series, 1994, pp. $35-42$.

[19] C. Rose, B. Guenter, B. Bodenheimer, and M. F. Cohen, "Efficient generation of motion transitions using spacetime constraints," in Proc. SIGGRAPH 96, Computer Graphics Annu. Conf. Series, 1996, pp. 147-154.

[20] M. Gleicher, "Motion editing with spacetime constraints," in Proc. 1997 Symp. Interactive 3-D Graphics, 1997, pp. 139-148.

[21] J. K. Hodgins and N. S. Pollard, "Adapting simulated behaviors for new characters," in Proc. SIGGRAPH 97, Computer Graphics Annu. Conf. Series, 1997, pp. 153-162.

[22] T. Komura and Y. Shinagawa, "A muscle-based feed-forward controller for the human body," Comput. Graph. Forum, vol. 16, no. 3, pp. 165-176, 1997.

[23] A. Bruderlin and L. Williams, "Motion signal processing," in Proc. SIGGRAPH 95, Computer Graphics Annu. Conf. Series, 1995, pp. 97-104.

[24] T. Flash and N. Hogan, "The coordination of arm movements: an experimentally confirmed mathematical model," J. Neurosci., vol. 5, pp. $1688-1703,1985$.

[25] Y. Uno, M. Kawato, and R. Suzuki, "Formation and control of optimal trajectory in human multijoint arm movement," Biolog. Cybern., vol. 61, pp. 89-101, 1989.
[26] M. Kawato, "Optimization and learning in neural networks for formation and control of coordinated movement," in Attention and Performance, D Meyer and S. Kornblum, Eds. Cambridge, MA: MIT Press, 1992, vol. XIV, pp. 821-849.

[27] C. M. Harris and D. M. Wolpert, "Signal-dependent noise determines motor planning," Nature, vol. 394, pp. 780-784, 1998.

[28] J. F. Soechting and C. A. Terzuolo, "Organization of arm movements. Motion is segmented," Neuroscience, vol. 23, pp. 39-51, 1987.

[29] — "Organization of arm movements in three-dimensional space. Wrist motion is piecewise planar," Neuroscience, vol. 23, pp. 53-61, 1987.

[30] F. Lacquaniti, C. A. Terzuolo, and P. Viviani, "The law relating the kinematic and figural aspects of drawing movements," Acta Psychologica vol. 54, pp. 115-130, 1983.

[31] F. E. Pollick and G. Sapiro, "Constant affine velocity predicts the 1/3 power law of planar motion perception and generation," Vis. Res., vol 37, pp. 347-353, 1996.

[32] F. E. Pollick, T. Flash, P. J. Giblin, and G. Sapiro, "Three-dimensional movements at constant affine velocity," Soc. Neurosci. Abstracts, vol 23, no. 2, p. 2237, 1997

[33] P. Viviani and N. Stucchi, "Biological movements look uniform: evidence of motor-perceptual interactions," J. Exp. Psych.: Human Perception Perform., vol. 18, pp. 602-623, 1992

[34] H. Hill and F. E. Pollick, "Exaggerating temporal differences enhances recognition of individuals from point light displays," Psych. Sci., vol. 11 , no. 3, pp. 223-228, 2000.

[35] F. E. Pollick, C. M. Fidopiastis, and V. Braden, "Recognizing the style of spatially exaggerated tennis serves," Perception, Perception, vol. 30 , pp. 323-338, 2001.

[36] M. A. Giese and T. Poggio, "Morphable models for the analysis and synthesis of complex motion patterns," Int. J. Comput. Vis., vol. 38, no. 1, pp. 59-73, 2000.

[37] M. A. Giese and M. Lappe, "Perception of generalization fields for the recognition of biological motion," Vis. Res., vol. 42, pp. 1847-1858, 2002.

[38] F. E. Pollick, H. Paterson, A. Bruderlin, and A. J. Sanford, "Perceiving affect from arm movement," Cognition, vol. 82, pp. B51-B61, 2001.

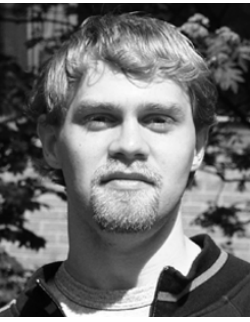

Joshua G. Hale received the B.A. and M.A. degrees in computation from Oxford University, Oxford, U.K., where he also won the C.A.R. Hoare Prize for Computation. He received the M.Sc. degree in computer science from Edinburgh University, Edinburgh, U.K., and the Ph.D. degree in motion synthesis from Glasgow University, Glasgow, U.K.

$\mathrm{He}$ is a Researcher in the Humanoid Robotics Group, JST-ICORP Computational Brain Project, ATR Computational Neuroscience Laboratories, Kyoto, Japan. His current work is focussed on dynamic simulation and motion generation for humanoid robots

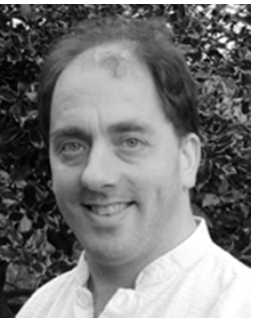

Frank E. Pollick received the B.S. degree physics and biology from the Massachussets Institute of Technology, Cambridge, the M.Sc. degree in biomedical engineering from Case Western Reserve University, Cleveland, $\mathrm{OH}$, and the $\mathrm{Ph} . \mathrm{D}$. degree in cognitive sciences from the University of California, Irvine, in 1991

He was a researcher at ATR Human Information Processing Research Laboratories in Kyoto, Japan from 1991 to 1997. Since 1997, he has been in the Department of Psychology, University of Glasgow, Glasgow, U.K. His research interests include the recognition of affect and inten from human movement, the neural mechanisms underlying these capabilities, and the design of human-humanoid interactions. 